\title{
Reliability Analysis via an Optimal Covariance Matrix Adaptation Evolution Strategy: Emphasis on Applications in Civil Engineering
}

\author{
Ali Kaveh¹, Seyed Mohammad Javadi ${ }^{1}$ Roya Mahdipour Moghanni ${ }^{1}$ \\ ${ }^{1}$ Centre of Excellence for Fundamental Studies in Structural Engineering, Iran University of Science and Technology, Narmak, \\ University St., Hengam St., Resalat Square, 13114-16846, Tehran, Iran \\ * Corresponding author, e-mail: alikaveh@iust.ac.ir
}

Received: 21 February 2020, Accepted: 14 March 2020, Published online: 17 April 2020

\begin{abstract}
In this paper, a reliability-based optimization approach is applied using a recently proposed CMA-ES with optimal covariance update and storage complexity. Cholesky-CMA-ES gives a significant increase in optimization speed and reduces the runtime complexity of the standard CMA-ES. The reliability index is the shortest distance between the surface of Limit-State Function (LSF) and the origin of the standard normal space. Hence, finding the reliability index can be expressed as a constrained optimization problem. To verify the concept and test the feasibility of this algorithm, several numerical examples consisting of mathematical and highly nonlinear civil engineering problems are investigated. The reliability indexes obtained agree reasonably well with reported values from some existing approximation methods and Monte Carlo simulation.
\end{abstract}

Keywords

reliability analysis, FORM, optimal CMA-ES, computational efficiency, uncertainty

\section{Introduction}

In recent decades, reliability is progressively becoming important for the analysis and design of civil engineering systems. Hydraulic structures, oil and gas pipelines, and structural optimization with uncertainties are some examples of the application of reliability analysis. In this regard, different methods have been proposed to improve the accuracy and convergence rate of reliability analysis. Reliability analysis requires the specification of uncertain quantities and their distributions via the Limit-State Function (LSF). First Order Reliability Method (FORM), second-order reliability method (SORM), Response Surface Method (RSM), and Simulation techniques (e.g., Monte Carlo Method and Subset simulation) are wellknown methods. In addition, approximation methods such as response surface have been recently successfully applied in reliability analysis [1-2]. Among these methods, due to the efficiency and simplicity, the moment methods are widely used in reliability analysis [3]. However, an increasing number of dimensions amplify the difficulty in computing the failure probability using the classical FORM and SORM.
Finding the reliability index can be expressed as a constrained optimization problem because the reliability index is the shortest distance between the surface of LSF and the origin of the standard normal space.

Recently, researchers have employed different mathematical and evolutionary algorithms for optimization in civil engineering such as [4-8]. In reliability engineering, $\mathrm{Fu}$ and Frangopol [9] proposed a framework to achieve minimum structural weight, highest system reliability, and highest system redundancy. Kaveh et al. [10] utilized the charged system search (CSS) algorithm as an optimization tool to achieve the minimum reliability index under the limit state function. In other applications of reliabilitybased optimization in civil engineering, Deshpande et al. proposed reliability-based optimization models for scheduling rehabilitation actions for flexible pavements [11]. Lógó et al. [12] proposed probabilistic topology optimization method and Several new topology design problems was calculated with randomly given loads. Csébfalvi [13] presented a new theoretical model and a problem-specific metaheuristic approach when the only source of 
uncertainty is the variability of the applied load directions. Kalatjari et al. [14] used improved algebraic force method and artificial intelligence for system reliability assessment of redundant trusses. Recently, Kaveh and Dadras [15] proposed an efficient method for reliability estimation using the combination of asymptotic sampling and weighted simulation.

In 2003, Hansen et al. [16] introduced a novel evolutionary optimization strategy based on the randomized evolution strategy with covariance matrix adaptation (CMA-ES). This derivative-free algorithm demonstrates good performance in numerous applications; however, the computational cost of CMA-ES is its main problem. Recently, Krause et al. [17] proposed a CholeskyCMA-ES method, which reduces the standard CMA-ES complexity. In addition, it reduces the memory footprint of the algorithm.

The purpose of the current study is to verify the concept and test the feasibility of this framework in the reliability analysis of highly nonlinear civil engineering problems.

The paper is structured as follows. Section 2 introduces the approximation concepts in reliability analysis. Section 3 presents the descriptions of Cholesky-CMA-ES. In Section 4, numerical examples are studied to show the efficiency and accuracy of the proposed method, and some concluding remarks are finally provided in Section 5.

\section{Approximation concepts in reliability analysis}

A typical limit-state function can be written as:

$g(R, S)=R-S$,

where $R$ is the resistance, and $S$ is the load. If we denote the failure event as $f=\{g<0\}$, then we can write:

Failure probability $=P_{f}=P(g(R, S)<0)$

$$
=P(R(X)-S(X)<0) .
$$

The exact evaluation of failure probability is not possible for most practical engineering problems because the limit state functions are usually highly nonlinear, and the number of parameters is usually large. On the other hand, in most cases, the limit state functions are implicit. The failure probability in correspondence with a reliability index $(\beta)$ is defined as follows:

$$
P_{f}=1-(\beta) \text {, }
$$

where $\Phi$ denotes cumulative distribution function (CDF) of standard normal distribution.

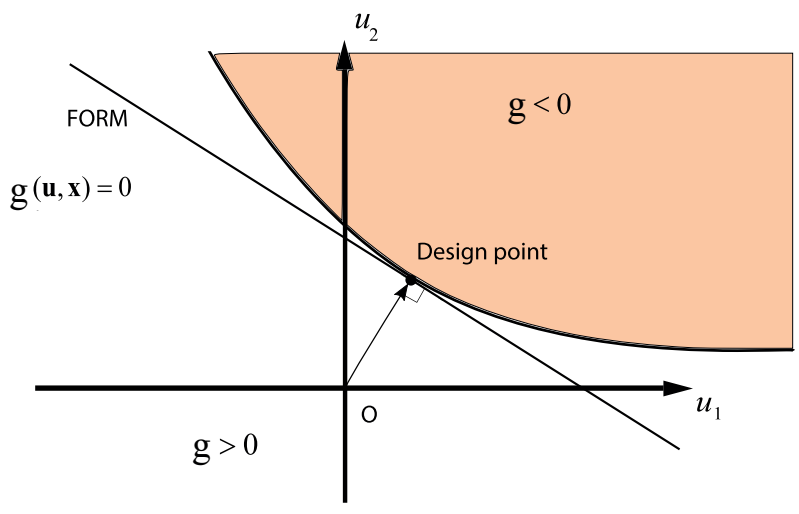

Fig. 1 Reliability index and relevant design point

As shown in Fig. 1, the reliability index is the shortest distance between the surface of LSF and the origin of the standard normal space [18]. Therefore, finding the design point can be expressed as a constrained optimization problem:

Find

$$
U=\left[u_{1}, u_{2}, \ldots, u_{n}\right],
$$

To minimize $\beta=\left(U^{T} U\right)^{\frac{1}{2}} \times f_{\text {penalty }}$,

Subject to: $\quad g\left(X_{i}, d_{k}\right)=0$,

where $u_{i}$ are the uncorrelated normalized random variables, $X_{i}$ is the random variable vector and $d_{k}$ are the deterministic design parameters. In this paper, the penalty function is utilized in order to make the problem unconstrained:

$$
f_{\text {penalty }}(X)=\left(1+\varepsilon_{1} \vartheta\right)^{\varepsilon_{2}}, \quad \vartheta=\sum_{i=1}^{p} \vartheta_{i},
$$

where $p$ is the total number of constraints. The values of $\vartheta_{i}$ are set to zero for satisfied constraints, while for violated constraints they are selected considering the severity of the violation. Here, $\varepsilon_{1}$ is set to unity, and $\varepsilon_{2}$ starts from 1.5 increases linearly to 4 in iterations for all numerical examples.

Normal distributions can be transformed into standard normal distributions by:

$u_{i}=\frac{x_{i}-\mu_{i}}{\sigma_{i}}$,

where $x_{i}$ is the ith component of $X, \mu_{i}$ and $\sigma_{i}$ are the mean value and the standard deviation of $x_{i}$, respectively. In this paper, the normal-tail approximation method is used in order to transform a lognormal distribution variable to an equivalent normal distribution variable. In this method, the cumulative distribution functions (CDF) and probability density function (PDF) of the original, non-normal random 
variable distribution, and the equivalent normal random variable distributions at the most probable failure point (MPP) are equal [19]. The mean and standard deviation of the equivalent normal variable are calculated as follows:

$$
\begin{gathered}
\sigma_{x^{\prime}}=\frac{\varphi\left(\Phi^{-1}\left[F_{x}\left(x^{*}\right)\right]\right)}{f_{x}\left(x^{*}\right)}, \\
\mu_{x^{\prime}}=x^{*}-\Phi^{-1}\left[F_{x}\left(x^{*}\right)\right] \sigma_{x^{\prime}},
\end{gathered}
$$

where $\mu_{x^{\prime}}$ and $\sigma_{x^{\prime}}$ are the equivalent means and standard deviations of the approximate normal distributions. In this equation $\varphi$ and $\Phi$ denote probability density function (PDF) and cumulative distribution function (CDF) of the standard normal distribution, respectively. $f_{x}\left(x^{*}\right)$ and $F_{x}\left(x^{*}\right)$ are the PDF and CDF of the original variable $X$, respectively, at the design point (i.e., at $x^{*}$ ).

\section{Cholesky-CMA-ES}

Covariance Matrix Adaptation Evolution Strategy (CMA-ES) [16] is a successful optimization algorithm. Similar to many evolutionary algorithms, CMA-ES has three parts: I: Mutation, which is used for exploration; II: Recombination for exploration, and III: Selection which is used for exploitation and convergence rate [20].

CMA-ES samples from a sequence of multivariate normal distributions. In this paper, the default strategy parameters and the CMA-ES algorithm is presented according to Hansen's tutorial paper [21].

One of the drawbacks of CMA-ES is the high computational cost of CMA-ES. Accordingly, Krause et al. proposed a Cholesky-CMA-ES to reach optimal time complexity. It allows for the numerically stable computation of the inverse of the Cholesky factor in quadratic time and provides the eigenvalues of the covariance matrix without additional costs [17]. To minimize Eq. (2), CholeskyCMA-ES is used as a powerful optimization method. The optimization process is outlined in Algorithms 1 and 2.

\section{Numerical examples}

In order to investigate the efficiency of the algorithm, some numerical examples are presented. In addition, this section discusses four engineering design problems, i.e., three-span continuous beam, one-bay one-story frame, concrete gravity dam, and primary-secondary. In order to handle constraints, the penalty function is used, which magnifies the weight of the infeasible solutions in order to make the problem unconstrained. All the computational

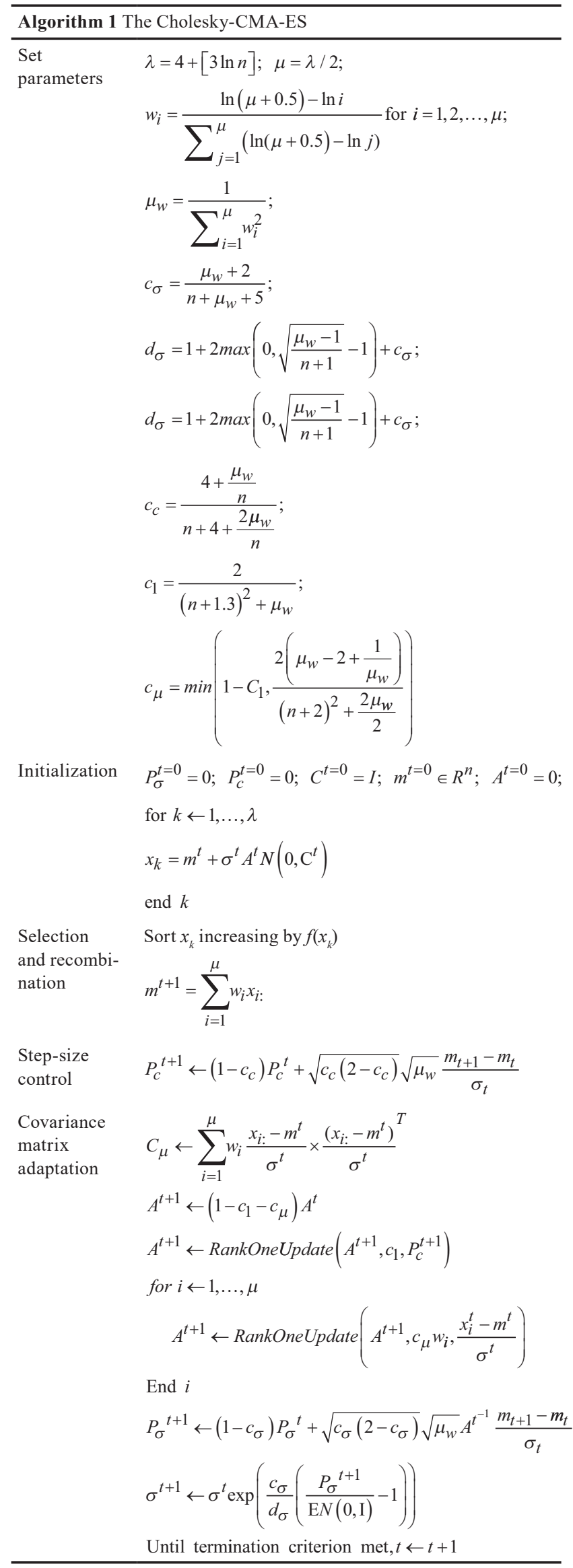




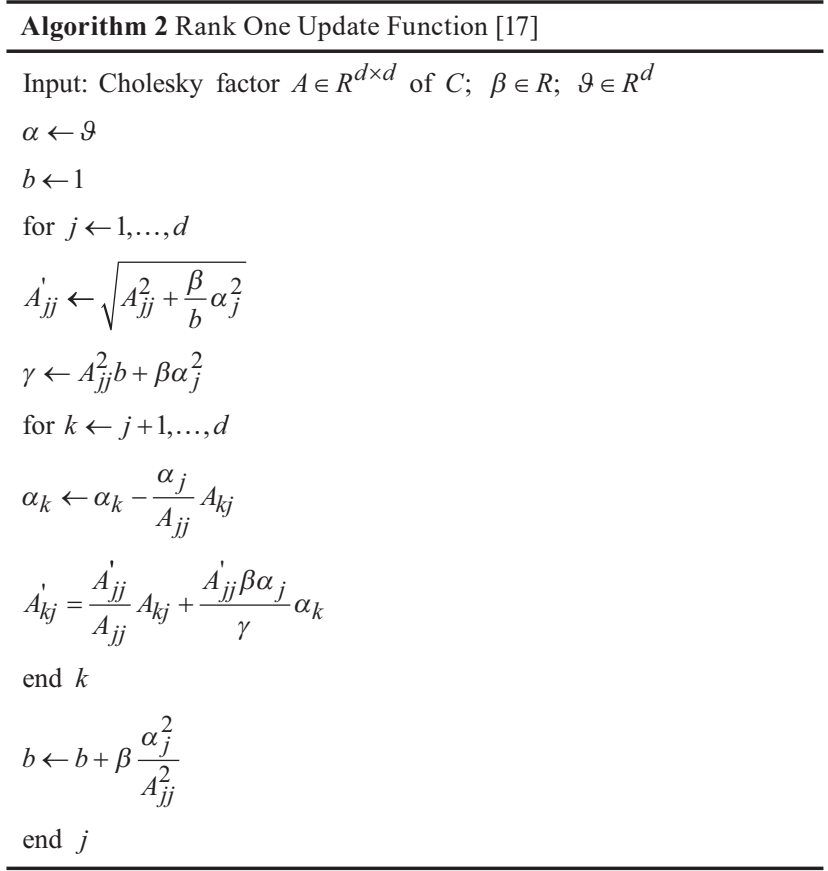

parts were performed utilizing the MATLAB software. Twenty independent optimization runs with 200 iterations are carried out for the considered examples.

\subsection{Mathematical examples}

In this section, the performance of the Cholesky-CMA-ES algorithm is investigated using three well-known numerical problems used in the literature. The results are then compared with FORM, Monte Carlo simulation (MCS), and results obtained by other algorithms.

\subsubsection{A cubic polynomial with mixed term}

This problem is proposed in [22]. The LSF of the first example is a cubic polynomial with mixed term as:

$g(X)=x_{1}^{3}+x_{1}^{2} x_{2}+x_{2}^{3}-18$

where $x_{1}$ and $x_{2}$ are independent standard normal distribution (with zero mean and unit standard). A comparison with the results of other references is provided in Table 1. The design point obtained from Cholesky-CMA-ES is $X^{*}=[-0.22368,2.61492]$.

\subsubsection{A highly nonlinear 10-variable LSF}

This example is proposed in [25]. The LSF is a highly nonlinear 10-variable and is defined as:

$g(X)=2+0.015\left(\sum_{i=1}^{9} x_{i}^{2}\right)^{3}-x_{10}$

Table 2 presents the statistics of random variables. The reference value for the probability of failure obtained by MCS using $10^{6}$ samples is $4 \times 10^{-6}(\beta=4.4651)$. Roudak et al. [26] solved the example resulting in $P_{f}=8.78 \times 10^{-5}$ $(\beta=3.7515)$. For this example, the optimal reliability index and the corresponding probability of failure obtained by Cholesky-CMA-ES are 4.78 and $8.79 \times 10^{-7}$, respectively. The design point obtained from the algorithm in original space is $X^{*}=[0.2935,0.3008,0.2547,0.2778,0.2832$, $0.2814,0.2703,0.2857,0.2498,2.0050]$.

\subsubsection{Series system problem}

This problem consists of a series system involving linear and convex LSFs in a two-dimensional standard Gaussian space [27].

$$
g(X)=\min \left\{\begin{array}{c}
0.1\left(x_{1}-x_{2}\right)^{2}-\frac{\left(x_{1}+x_{2}\right)}{\sqrt{2}}+3 \\
0.1\left(x_{1}-x_{2}\right)^{2}+\frac{\left(x_{1}+x_{2}\right)}{\sqrt{2}}+3 \\
x_{1}-x_{2}+\frac{7}{\sqrt{2}} \\
x_{2}-x_{1}+\frac{7}{\sqrt{2}}
\end{array}\right.
$$

The optimal reliability index and the corresponding probability of failure obtained by crude Monte-Carlo, standard deterministic finite element code [24], Adaptive radial-based importance sampling method [25], ECBO [26]

Table 2 The statistics of random variables

\begin{tabular}{lccc}
\hline Variables & Distribution & Mean & Standard deviation \\
\hline$x_{1}, x_{2}, \ldots, x_{10}$ & Normal & 1 & 0.5 \\
\hline
\end{tabular}

Table 1 Comparison for the cubic polynomial with mixed term

\begin{tabular}{lcccccccc}
\hline & $\begin{array}{c}\text { MCS } \\
\text { (106 samples) }\end{array}$ & FORM & $\begin{array}{c}\text { Wang and } \\
\text { Grandhi [22] }\end{array}$ & $\begin{array}{c}\text { Gong et al. } \\
{[23]}\end{array}$ & $\begin{array}{c}\text { Keshtegar } \\
{[24]}\end{array}$ & Yang [25] & $\begin{array}{c}\text { Roudak et al. } \\
{[26]}\end{array}$ & Present work \\
\hline $\begin{array}{l}\text { Reliability } \\
\text { index }\end{array}$ & 2.5274 & 2.32 & 2.2983 & 2.2983 & 2.2982 & 2.298 & 2.2983 & 2.5107 \\
$\begin{array}{l}\text { The probability } \\
\text { of failure }\end{array}$ & 0.0057 & 0.0102 & 0.0108 & 0.0108 & 0.0108 & 0.0108 & 0.0108 & 0.006 \\
\hline
\end{tabular}


Table 3 Comparison for the series system problem

\begin{tabular}{lcccc}
\hline & MCS & Borri and Speranzini [28] & Grooteman [29] & $\begin{array}{c}\text { Kaveh and Ilchi } \\
\text { Ghazaan (ECBO) [30] }\end{array}$ \\
\hline Reliability index & 3 & 2.9999 & 2.925 & 2.9999 \\
The probability of failure & 0.0013 & 0.0014 & 0.0017 & 0.0014 \\
Design point in original space & - & $X^{*}=[2.1212,2.1212]$ & - & $X^{*}=[-2.1201,-2.1223]$ \\
\hline
\end{tabular}

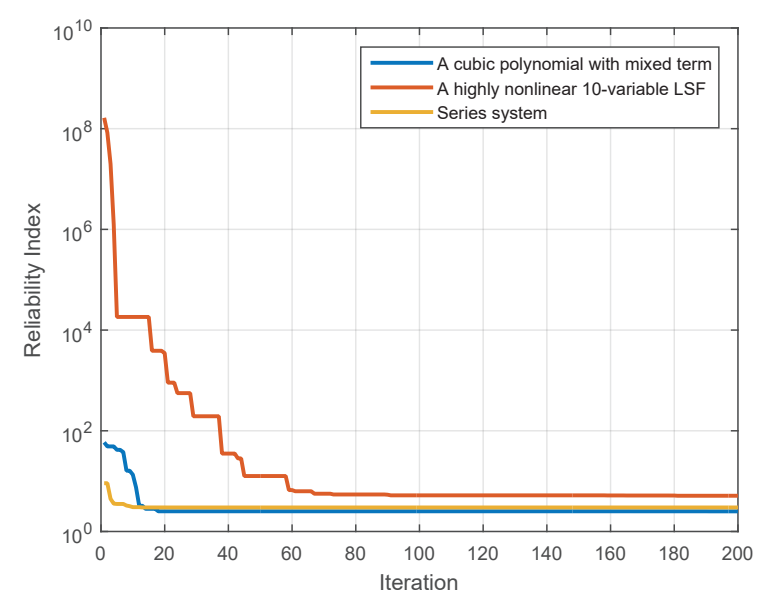

Fig. 2 The best convergence curves of reliability index for mathematical examples (semi-log plot)

and proposed algorithms are shown in Table 3. The convergence histories of Cholesky-CMA-ES for mathematical examples are illustrated in Fig. 2. Comparisons between MCS as an exact method with Cholesky-CMA-ES show the robustness of the algorithm.

\subsection{Engineering design problems}

In this section, five well-known engineering design problems used in the literature have been investigated. The results are then compared with Monte Carlo simulation (MCS) and the results obtained by other algorithms.

\subsubsection{Three-span continuous beam}

Consider a three-span continuous beam shown in Fig. 3. The maximum vertical deflection of the beam determines the LSF:

$g(w, E, h)=\frac{L}{360}-360 \frac{w}{E h^{4}}$

where $W, L, E$, and $h$ are the intensity of the uniform gravity load, span length, modulus of elasticity, and height of the section, respectively. The statistics of the variables are listed in Table 4. Table 5 summarizes the estimates of the reliability index and probability of failure based on MCS, Roudak et al. [26], and the present work.

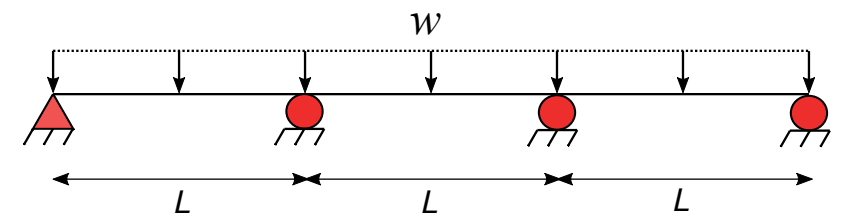

Fig. 3 Three-span continuous beam

Table 4 Description of random variables in the three-span continuous beam

\begin{tabular}{lccc}
\hline Variable & Distribution & Mean & Standard deviation \\
\hline$W(\mathrm{kN} / \mathrm{m})$ & Normal & 10 & 0.4 \\
$L(\mathrm{~m})$ & Deterministic & 7 & 0 \\
$E\left(\mathrm{kN} / \mathrm{m}^{2}\right)$ & Normal & $2 \times 10^{7}$ & $0.5 \times 10^{7}$ \\
$h(\mathrm{~m})$ & Normal & 0.4 & 0.01 \\
\hline
\end{tabular}

Table 5 Comparison for the three-span continuous beam

\begin{tabular}{cccc}
\hline & $\begin{array}{c}\text { MCS } \\
\left(10^{6} \text { samples }\right)\end{array}$ & $\begin{array}{c}\text { Roudak } \\
\text { et al. [26] }\end{array}$ & Present work \\
\hline $\begin{array}{c}\text { Reliability } \\
\text { index }\end{array}$ & 3.4765 & 2.5217 & 2.91 \\
$\begin{array}{c}\text { The probability } \\
\text { of failure }\end{array}$ & $2.54 \times 10^{-4}$ & $5.8 \times 10^{-3}$ & $1.8 \times 10^{-3}$ \\
$\begin{array}{c}\text { Design point in } \\
\text { original space }\end{array}$ & - & - & $\begin{array}{r}X^{*}=[10.5474, \\
\left.8.63 \times 10^{6}, 0.3878\right]\end{array}$ \\
\hline
\end{tabular}

\subsubsection{One-bay one-story frame}

This example is proposed in [31]. The failure is assumed to be described by first-order rigid-plastic hinge theory. The structure is subjected to static loads, including a horizontal load $P_{1}$ and a vertical load $P_{2}$. Accordingly, there are three relevant collapse mechanisms, as shown in Fig. 4. The LSF for these failure modes are derived as:

$h_{1}(X)=4 \frac{M_{p l}}{L}-P_{1}$

$h_{2}(X)=4 \frac{M_{p l}}{L}-P_{2}$

$h_{3}(X)=6 \frac{M_{p l}}{L}-P_{1}-P_{2}$

$X=\left[P_{1}, P_{2}\right]$. 

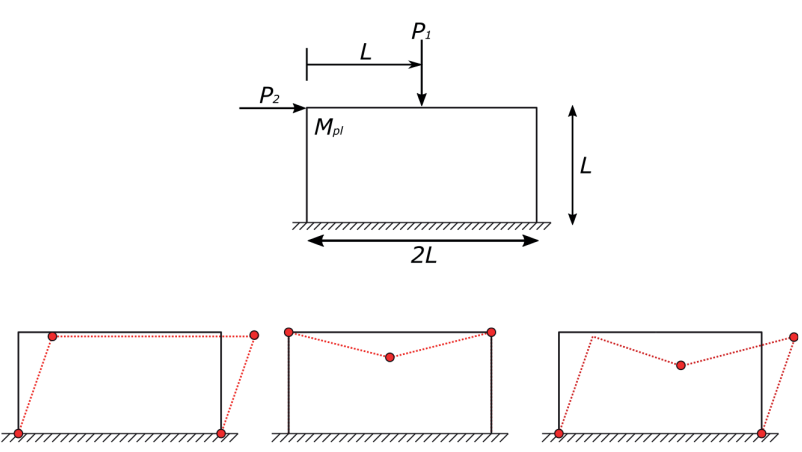

Fig. 4 One-bay one-story frame

It is assumed that the plastic moment $M_{p l}$ is deterministic. The failure of the system occurs if just one of the components fails. Hence, this problem is a series system with three components. The limit state in the standard Gaussian space is shown in Fig. 5. Table 6 and Table 7 present the statistics of random variables and the results, respectively. The design point obtained from Cholesky-CMA-ES in the original space is $X^{*}=[3.0255,2.9742]$.

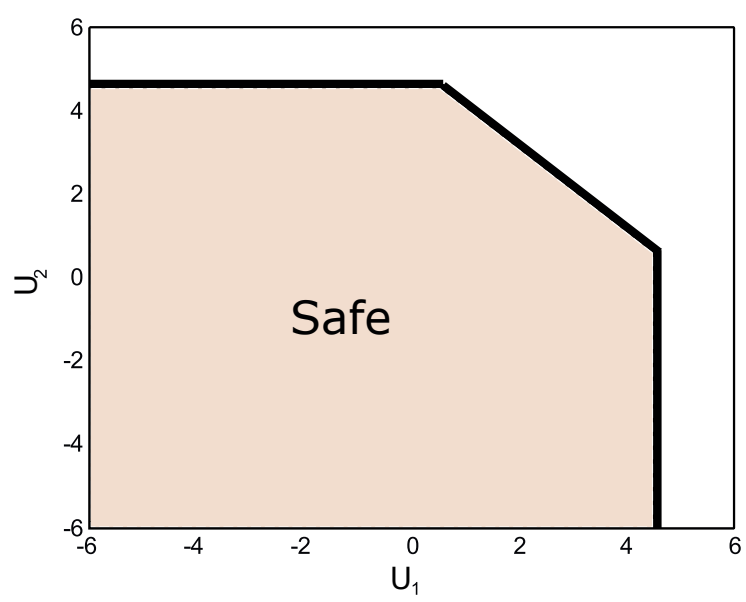

Fig. 5 Limit state in the standard Gaussian space

Table 6 Description of random variables in the one-bay one-story frame

\begin{tabular}{lccc}
\hline Variable & Distribution & Mean & Coefficient of Variation \\
\hline$P_{1}$ & Normal & $1.7 \frac{M_{p l}}{L}$ & 0.5 \\
$P_{2}$ & Normal & $1.7 \frac{M_{p l}}{L}$ & 0.5 \\
\hline
\end{tabular}

Table 7 Comparison for the one-bay one-story frame

\begin{tabular}{lccc}
\hline & $\begin{array}{c}\text { MCS } \\
\left(10^{6} \text { samples }\right)\end{array}$ & $\begin{array}{c}\text { Subset } \\
\text { Simulation [32] }\end{array}$ & Present work \\
\hline $\begin{array}{l}\text { Reliability } \\
\text { index }\end{array}$ & 3.69 & 3.86 & 3.68 \\
$\begin{array}{l}\text { The probability } \\
\text { of failure }\end{array}$ & $1.11 \times 10^{-4}$ & $5.66 \times 10^{-5}$ & $1.16 \times 10^{-4}$ \\
\hline
\end{tabular}

4.2.3 The anti-slide reliability of a concrete gravity dam This example is a concrete gravity dam shown in Fig. 6. The limit state function is defined by [33]:

$$
\begin{aligned}
& g\left(\alpha, h, f^{\prime}, c^{\prime}\right)=f^{\prime}\left(\sum W-U\right)+c^{\prime} T-\sum P \\
& =f^{\prime}\left(3.096 \times 10^{7}-2.156 \times 10^{5} a h-2.744 \times 10^{4} h\right) \\
& +44 c^{\prime}-4900 h^{2}=0,
\end{aligned}
$$

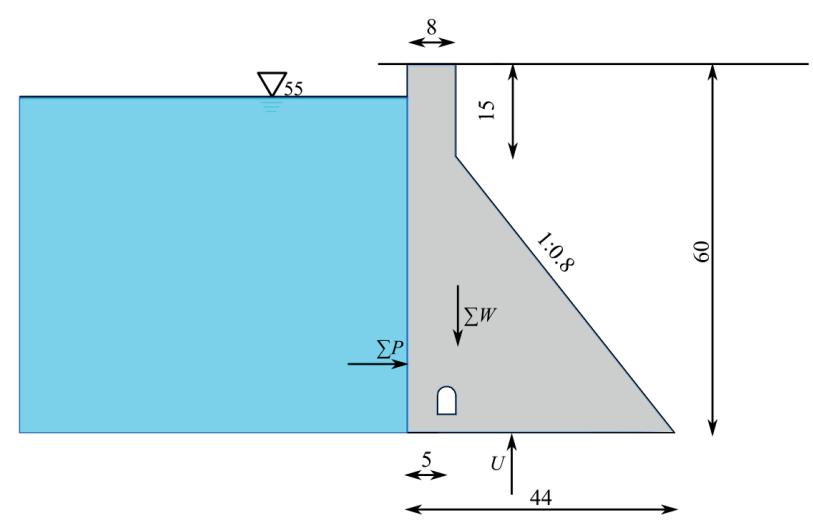

Fig. 6 The anti-slide reliability of a concrete gravity dam

where $\mu_{h}=0.935 \mathrm{H}-0.33$. In this equation $W, U$, and $P$ are the weight of dam, uplift pressure, and hydrostatic pressure, respectively. The material density is $\rho=2400 \mathrm{~kg} / \mathrm{m}^{3}$, and the statistics of these variables are given in Table 8 . The optimal reliability index achieved by ABC optimization method [33] is $5.51\left(P_{f}=1.79 \times 10^{-8}\right)$. While the reference value for the probability of failure obtained by MCS using $10^{6}$ samples is $0.0069(\beta=4.4651)$. For this example, the optimal reliability index and the corresponding probability of failure obtained by Cholesky-CMA-ES are 3.89 and $4.96 \times 10^{-5}$ respectively. The design point obtained from Cholesky-CMA-ES in original space is $X^{*}=[0.0517$, $56.3663,0.5408,0.9985]$.

Table 8 Description of Random Variables in the anti-slide reliability of a concrete gravity dam

\begin{tabular}{lcccc}
\hline Description & Variables & Distribution & Mean & $\begin{array}{l}\text { Standard } \\
\text { deviation }\end{array}$ \\
\hline $\begin{array}{l}\text { The reduction factor } \\
\text { of uplift pressure }\end{array}$ & $\alpha$ & Gaussian & 0.25 & 0.075 \\
$\begin{array}{l}\text { The upstream water } \\
\text { level }\end{array}$ & $\mathrm{h}^{(1)}[\mathrm{m}]$ & Gaussian & 51.1 & 3.22 \\
$\begin{array}{l}\text { The shear friction } \\
\text { factor }\end{array}$ & $f^{\prime}$ & Gaussian & 1 & 0.2 \\
$\begin{array}{l}\text { The shear cohesion } \\
\text { The }\end{array}$ & $c^{\prime}$ & Lognormal & 0.9 & 0.36 \\
\hline
\end{tabular}

(1) $\mathrm{H}=55$ 


\subsubsection{Reliability of the uncertain primary-secondary system}

This example is originally presented in Kiureghian and Stefano [34]. The two-degree-of-freedom primary-secondary system shown in Fig. 7. Igusa and Der Kiureghian

[35] proved that the mean-square relative displacement

response of the secondary spring to a white-noise base excitation of the system can be written as [34]:

$E\left(x_{s}^{2}\right)=\frac{\pi S_{0}}{4 \zeta_{s} \omega_{s}^{3}}\left[\frac{\zeta_{a} \zeta_{s}}{\zeta_{p} \zeta_{s}\left(4 \zeta_{a}^{2}+\eta^{2}\right)+\vartheta \zeta_{a}^{2}} \frac{\left(\zeta_{p} \omega_{p}^{3}+\zeta_{s} \omega_{s}^{3}\right) \omega_{p}}{4 \zeta_{a} \omega_{a}^{4}}\right]$

where $\omega_{p}=\sqrt{\frac{K_{p}}{M_{p}}} ; \omega_{s}=\sqrt{\frac{K_{s}}{M_{s}}} ; \omega_{a}=\frac{\left(\omega_{p}+\omega_{s}\right)}{2} ; \zeta_{a}=\frac{\left(\zeta_{p}+\zeta_{s}\right)}{2}$;

$\eta=\frac{\left(\omega_{p}-\omega_{s}\right)}{\omega_{a}}$ and $\vartheta=\frac{m_{s}}{m_{p}}$.

$p$ and $s$ denote the primary and secondary oscillators, respectively. The failure domain is defined as:

$g=F_{s}-K_{s} P \sqrt{\left[E\left(x_{s}^{2}\right)\right]}$.

In this equation, $P$ is a deterministic peak factor considered constant equal to 3 . The statistical properties of random variables are presented in Table 9. The optimal reliability index and the corresponding probability of failure obtained by researchers are listed in Table 10. The Cholesky-CMA-ES reached $\beta=2.1239$ and the $X^{*}$ equal to: $[1.0200,0.0102,1.0240,0.0104,0.0265,0.0113,13.5175$, $103.9595]$.
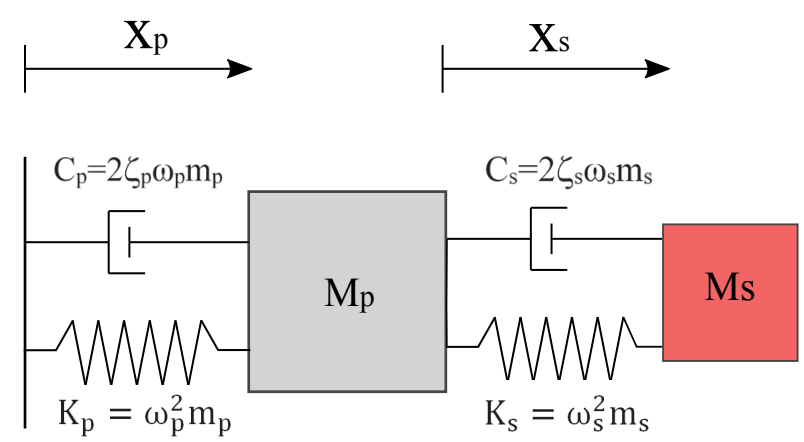

Fig. 7 A primary-secondary system
Table 9 Description of random variables in the primary-secondary dynamic system

\begin{tabular}{lccc}
\hline Variables & Distribution & Mean & Standard deviation \\
\hline$m_{p}$ & Lognormal & 1 & 0.1 \\
$m_{s}$ & Lognormal & 0.01 & 0.001 \\
$K_{p}$ & Lognormal & 1 & 0.2 \\
$K_{s}$ & Lognormal & 0.01 & 0.002 \\
$\zeta_{p}$ & Lognormal & 0.05 & 0.02 \\
$\zeta_{s}$ & Lognormal & 0.02 & 0.01 \\
$F_{s}$ & Lognormal & 15 & 1.5 \\
$S_{0}$ & Lognormal & 100 & 10 \\
\hline
\end{tabular}

\subsubsection{Reliability of the Three-bay, 12-story frame}

Fig. 8 shows the schematic and the loading conditions of the linear portal frame structure with twelve stories and three bays. Frame members are collected in 5 groups ( 3 column groups and 2 beam groups) consisting of 84 members. The sectional moments of inertia are expressed as [36]:

$I_{i}=\alpha_{i} A_{i}^{2}$

where $\alpha_{1}=\alpha_{2}=\alpha_{3}=0.08333 ; \alpha_{4}=0.2667 ; \alpha_{5}=0.2$,

In this example, the implicit LSF corresponds to the horizontal displacement of node " $\mathrm{A}$ " is defined as:

$g=0.096-U_{A}$.

The cross-sectional areas $A_{i}$ and horizontal load $P$ are random variables. The Young's modulus $\mathrm{E}$ is treated as deterministic and considered equal to $2.0 \times 10^{7} \mathrm{kN} / \mathrm{m}^{2}$.

Table 11 and Table 12 present the statistics of random variables and the results, respectively. The design point obtained from Cholesky-CMA-ES in the original space is $X^{*}=[0.244,0.160,0.350,0.191,0.147,40.01]$.

Fig. 9 provides convergence rates of engineering design problems. Comparisons between results show the accuracy and numerical performance of the algorithm.

\section{Conclusions}

In this paper, a newly-developed approximate covariance matrix adaptation evolution strategy, named as CholeskyCMA-ES, is applied to reliability analysis of some civil engineering problems.

Table 10 Comparison for the primary-secondary system

\begin{tabular}{lccccc}
\hline & MCS (10 samples) & Kiureghian and Stefano [34] & Keshtegar [24] & Roudak et al. [26] & Present work \\
\hline Reliability index & 2.737 & 2.12 & 2.0163 & 2.1231 \\
The probability of failure & 0.0031 & 0.017 & 0.0219 & 0.1239 \\
\hline
\end{tabular}



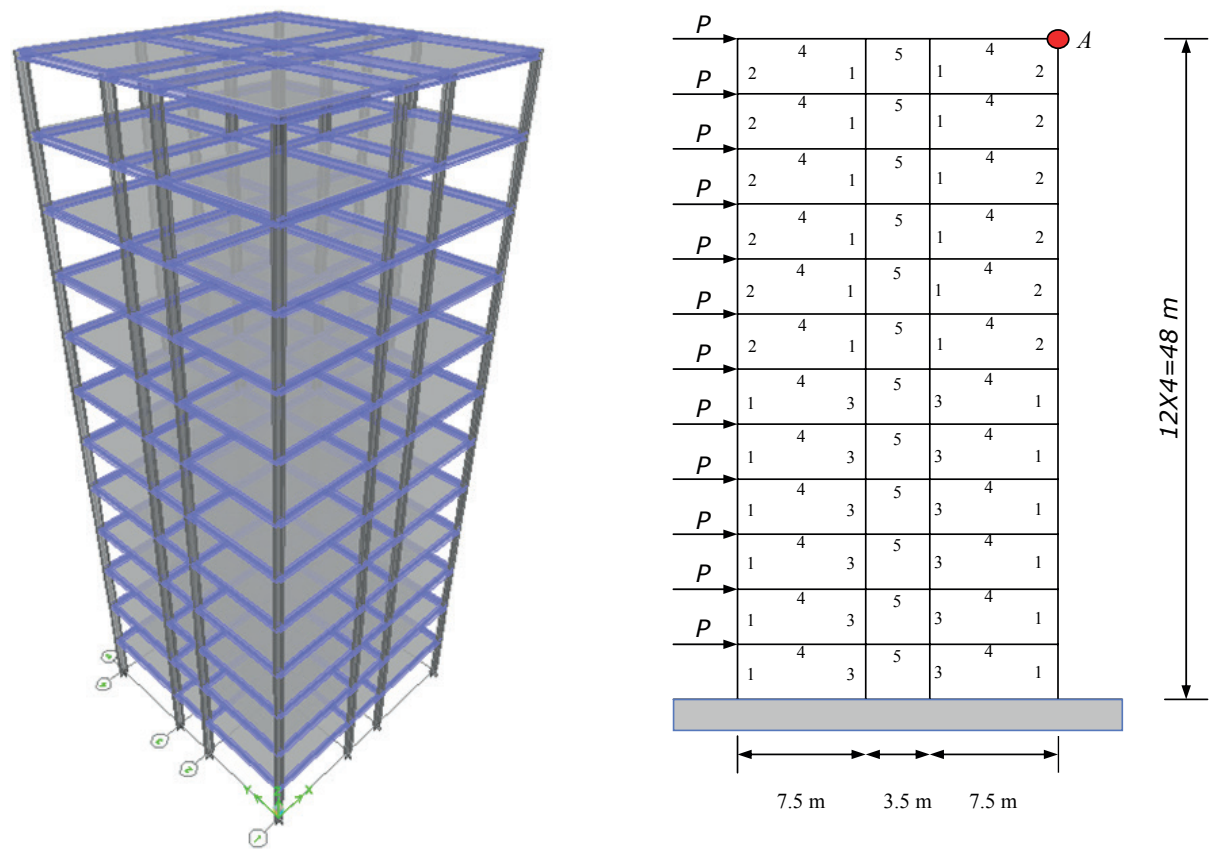

Fig. 8 A portal frame structure with twelve stories and three bays.

Table 11 Description of random variables in the portal frame structure

\begin{tabular}{lccc}
\hline Variables & Distribution & Mean & Standard deviation \\
\hline$A_{1}\left(\mathrm{~m}^{2}\right)$ & Lognormal & 0.25 & 0.025 \\
$A_{2}\left(\mathrm{~m}^{2}\right)$ & Lognormal & 0.16 & 0.016 \\
$A_{3}\left(\mathrm{~m}^{2}\right)$ & Lognormal & 0.36 & 0.036 \\
$A_{4}\left(\mathrm{~m}^{2}\right)$ & Lognormal & 0.20 & 0.020 \\
$A_{5}\left(\mathrm{~m}^{2}\right)$ & Lognormal & 0.15 & 0.015 \\
$\mathrm{P}(\mathrm{KN})$ & Type I Largest & 30 & 7.5 \\
\hline
\end{tabular}

Table 12 Comparison for the portal frame structure

\begin{tabular}{ccccc}
\hline & $\begin{array}{c}\text { MCS (105 } \\
\text { samples) }\end{array}$ & $\begin{array}{c}\text { Cheng [36]: } \\
\text { GA-ANN }\end{array}$ & $\begin{array}{c}\text { Cheng [36]: } \\
\text { GA-ANN-MCSIS }\end{array}$ & $\begin{array}{c}\text { Present } \\
\text { work }\end{array}$ \\
\hline $\begin{array}{c}\text { Reliability } \\
\text { index }\end{array}$ & 1.439 & 1.463 & 1.421 & 1.459 \\
$\begin{array}{c}\text { The } \\
\text { probability } \\
\text { of failure }\end{array}$ & 0.0751 & 0.0718 & 0.0777 & 0.0723 \\
\hline
\end{tabular}

In this algorithm, the original time and space complexity of CMA-ES are decreased using a quadratic-time covariance matrix update scheme with minimal memory requirements based on keeping triangular Cholesky factors. This algorithm is used to deal with some highly nonlinear reliability analysis problems.

The application to several mathematical and practical numerical examples with different type of random variables shows that the proposed framework provides accurate results. The reliability indexes obtained agree reasonably well with reported values from some existing

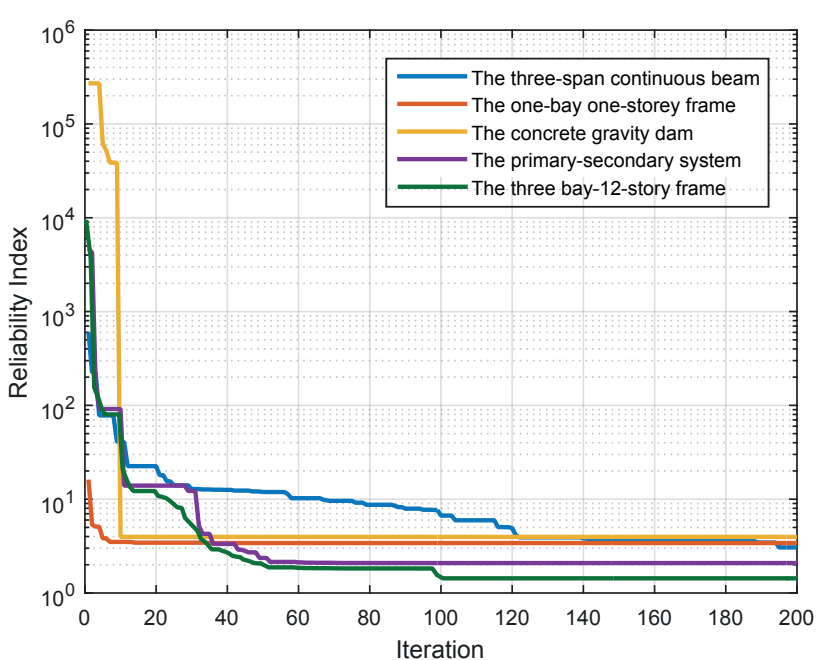

Fig. 9 The best convergence curves of reliability index for engineering design problems (semi-log plot)

approximation methods and Monte Carlo simulation. Therefore, this is general and suitable for different reliability engineering problems. Future research on this topic could be pursued in several areas. For instance, it is worth investigating the feasibility and the computational performance of other optimization algorithms, such as BIPOP-CMA-ES.

\section{Conflict of interest}

On behalf of all authors, the corresponding author states that there is no conflict of interest. 


\section{References}

[1] Faravelli, L. "Response-surface approach for reliability analysis", Journal of Engineering Mechanics, 115(12), pp. 2763-2781, 1989. https://doi.org/10.1061/(ASCE)0733-9399(1989)115:12(2763)

[2] Bucher, C. G., Bourgund, U. "A fast and efficient response surface approach for structural reliability problems", Structural Safety, 7(1), pp. $57-66,1990$. https://doi.org/10.1016/0167-4730(90)90012-E

[3] Aoues, Y., Chateauneuf, A. "Benchmark study of numerical methods for reliability-based design optimization", Structural and Multidisciplinary Optimization, 41(2), pp. 277-294, 2010. https://doi.org/10.1007/s00158-009-0412-2

[4] Hasançebi, O., Kazemzadeh Azad, S. "Adaptive dimensional search: A new metaheuristic algorithm for discrete truss sizing optimization", Computers \& Structures, 154, pp. 1-16, 2015.

https://doi.org/10.1016/j.compstruc.2015.03.014

[5] Kaveh, A., Moghanni, R. M., Javadi, S. M. "Ground Motion Record Selection Using Multi-objective Optimization Algorithms: A Comparative Study", Periodica Polytechnica Civil Engineering, 63(3), pp. 812-822, 2019.

https://doi.org/10.3311/PPci.14354

[6] Kazemzadeh Azad, S., Bybordiani, M., Kazemzadeh Azad, S., Jawad, F. K. J. "Simultaneous size and geometry optimization of steel trusses under dynamic excitations", Structural and Multidisciplinary Optimization, 58(6), pp. 2545-2563, 2018.

https://doi.org/10.1007/s00158-018-2039-7

[7] Kaveh, A., Bakhshpoori, T. "Metaheuristics: Outlines, MATLAB Codes and Examples", Springer, Cham, Switzerland, 2019. https://doi.org/10.1007/978-3-030-04067-3

[8] Kaveh, A. "Applications of metaheuristic optimization algorithms in civil engineering", Springer, Cham, Switzerland, 2017. https://doi.org/10.1007/978-3-319-48012-1

[9] Fu, G., Frangopol, D. M. "Balancing weight, system reliability and redundancy in a multiobjective optimization framework", Structural Safety, 7(2-4), pp. 165-175, 1990. https://doi.org/10.1016/0167-4730(90)90066-X

[10] Kaveh, A., Massoudi, M. S., Bagha, M. G. "Structural reliability analysis using charged system search algorithm", Iranian Journal of Science and Technology, 38(C2), pp. 439-448, 2014. https://doi.org/10.22099/IJSTC.2014.2420

[11] Deshpande, V. P., Damnjanovic, I. D., Gardoni, P. "Reliability-Based Optimization Models for Scheduling Pavement Rehabilitation", Computer-Aided Civil and Infrastructure Engineering, 25(4), pp. 227-237, 2010. https://doi.org/10.1111/j.1467-8667.2009.00636.x

[12] Lógó, J., Ghaemi, M., Rad, M. M. "Optimal Topologies in Case of Probabilistic Loading: The Influence of Load Correlation", Mechanics Based Design of Structures and Machines, 37(3), pp. 327-348, 2009. https://doi.org/10.1080/15397730902936328

[13] Csébfalvi, A. "A New Theoretical Approach for Robust Truss Optimization with Uncertain Load Directions", Mechanics Based Design of Structures and Machines, 42(4), pp. 442-453, 2014. https://doi.org/10.1080/15397734.2014.880064
[14] Kalatjari, V., Kaveh, A., Mansoorian, P. "System reliability assessment of redundant trusses using improved algebraic force method and artificial intelligence", Asian Journal of Civil Engineering, 12(4), pp. 523-550, 2011. [online] Available at: https://www.sid.ir/ en/Journal/ViewPaper.aspx?ID=248039 [Accessed: 10 March 2020]

[15] Kaveh, A., Dadras, A. "An efficient method for reliability estimation using the combination of asymptotic sampling and weighted simulation", Scientia Iranica, 26(4), pp. 2108-2122, 2019. https://doi.org/10.24200/SCI.2019.21367

[16] Hansen, N., Müller, S. D., Koumoutsakos, P. "Reducing the Time Complexity of the Derandomized Evolution Strategy with Covariance Matrix Adaptation (CMA-ES)", Evolutionary Computation, 11(1), pp. 1-18, 2003. https:/doi.org/10.1162/106365603321828970

[17] Krause, O., Arbonès, D. R., Igel, C. "CMA-ES with optimal covariance update and storage complexity", In: proceedings of the Neural Information Processing Systems (NIPS 2016), Barcelona, Spain, 2016, pp. 370-378.

[18] Hasofer, A. M., Lind, M. C. "An exact and invariant first order reliability format", Journal of Engineering Mechanics, 100(1), pp. 111-121, 1974.

[19] Rosenblatt, M. "Remarks on a Multivariate Transformation", The Annals of Mathematical Statistics, 23(3), pp. 470-472, 1952.

https://doi.org/10.1214/aoms/1177729394

[20] Omidvar, M. N., Li, X. "A Comparative Study of CMA-ES on Large Scale Global Optimization", In: Proceedings of the 23rd Australasian Joint Conference, Adelaide, Australia, Dec. 7-10, 2010, pp. 303-312. https://doi.org/10.1007/978-3-642-17432-2_31

[21] Hansen, N. "The CMA evolution strategy: A tutorial", Inria, ParisSaclay University, Paris, France, 2016. [online] Available at: https:// arxiv.org/abs/1604.00772 [Accessed: 10 March 2020]

[22] Wang, L., Grandhi, R. V. "Safety index calculation using intervening variables for structural reliability analysis", Computers \& Structures, 59(6), pp. 1139-1148, 1996. https://doi.org/10.1016/0045-7949(96)00291-X

[23] Gong, J. X., Yi, P., Zhao, N. "Non-Gradient-Based Algorithm for Structural Reliability Analysis", Journal of Engineering Mechanics, 140(6), Article number: 04014029, 2014. https://doi.org/10.1061/(ASCE)EM.1943-7889.0000722

[24] Keshtegar, B. "Chaotic conjugate stability transformation method for structural reliability analysis", Computer Methods in Applied Mechanics and Engineering, 310, pp. 866-885, 2016. https://doi.org/10.1016/j.cma.2016.07.046

[25] Yang, D. "Chaos control for numerical instability of first order reliability method", Communications in Nonlinear Science and Numerical Simulation, 15(10), pp. 3131-3141, 2010. https://doi.org/10.1016/j.cnsns.2009.10.018

[26] Roudak, M. A., Shayanfar, M. A., Barkhordari, M. A., Karamloo, M. "A robust approximation method for nonlinear cases of structural reliability analysis", International Journal of Mechanical Sciences, 133, pp. 11-20, 2017. https://doi.org/10.1016/j.ijmecsci.2017.08.038 
[27] Waarts, P. H. "Structural reliability using finite element analysis-An appraisal of DARS: Directional Adaptive Response surface Sampling", PhD thesis, TU Delft, Delft, The Netherlands, 2000. [online] Available at: http://resolver.tudelft.nl/uuid:6e6d9a76-fd124220-9dc1-36515b3f638d [Accessed: 10 March 2020]

[28] Borri, A., Speranzini, E. "Structural reliability analysis using a standard deterministic finite element code", Structural Safety, 19(4), pp. 361-382, 1997.

https://doi.org/10.1016/S0167-4730(97)00017-9

[29] Grooteman, F. "Adaptive radial-based importance sampling method for structural reliability", Structural Safety, 30(6), pp. 533-542, 2008. https://doi.org/10.1016/j.strusafe.2007.10.002

[30] Kaveh, A., Ilchi Ghazaan, M. "Structural reliability assessment utilizing four metaheuristic algorithms", International Journal of Optimization in Civil Engineering, 5(2), pp. 205-225, 2015. [online] Available at: http://ijoce.iust.ac.ir/article-1-210-en.html [Accessed: 10 March 2020]

[31] Bucher, C. "Computational Analysis of Randomness in Structural Mechanics", CRC Press, Leiden, The Netherlands, 2009.
[32] Li, H.-S., Cao, Z.- J. "Matlab codes of Subset Simulation for reliability analysis and structural optimization", Structural and Multidisciplinary Optimization, 54(2), pp. 391-410, 2016. https://doi.org/10.1007/s00158-016-1414-5

[33] Li, H., Li, J., Kang, F. "Artificial Bee Colony Algorithm for Reliability Analysis of Engineering Structures", Advanced Materials Research, 163-167, pp. 3103-3109, 2011.

https://doi.org/10.4028/www.scientific.net/AMR.163-167.3103

[34] Kiureghian, A. D., De Stefano, M. "Efficient Algorithm for SecondOrder Reliability Analysis", Journal of Engineering Mechanics, 117(12), pp. 2904-2923, 1991. https://doi.org/10.1061/(ASCE)0733-9399(1991)117:12(2904)

[35] Igusa, T., Der Kiureghian, A. "Dynamic Characterization of TwoDegree-of-Freedom Equipment-Structure Systems", Journal of Engineering Mechanics, 111(1), pp. 1-9, 1985. https://doi.org/10.1061/(ASCE)0733-9399(1985)111:1(1)

[36] Cheng, J. "Hybrid genetic algorithms for structural reliability analysis", Computers \& Structures, 85(19-20), 1524-1533, 2007. https://doi.org/10.1016/j.compstruc.2007.01.018 